\title{
Spontaneous Orbital Hematoma Induced by VALSAVA Maneuver
}

\author{
N. Inzale ${ }^{1 *}$, D. Jaafari ${ }^{1}$, H. Atidi ${ }^{1}$, S. Boutgayout ${ }^{1}$, S. Bleghmaidi ${ }^{1}$, I. Hajji ${ }^{1}$, A. Moutaouakil ${ }^{1}$
}

Ophthalmology Department, CHU Mohammed VI - Marrakesh, Morocco

DOI: $10.36347 /$ sjmcr.2021.v09i04.028

| Received: 18.03.2021 | Accepted: 24.04.2021 | Published: 27.04.2021

*Corresponding author: N. Inzale

Abstract

Case Report

Spontaneous intra-orbital hematoma is a rare, potentially serious condition, rapidly endangering the visual prognosis by affecting the optic nerve. It associates in its typical form a painful exophthalmia of brutal installation and oculomotor problems. Its etiologies are numerous and its diagnosis in the absence of an evocative context, relies mainly on anamnestic and radiological findings, particularly those provided by angio-MRI. This article illustrates a case of intra-orbital hematoma induced by the Valsalva maneuver, highlighting the fundamental role of anamnestic, clinical and radiological investigation in the etiological diagnosis of this pathology.

Keywords: Spontaneous Orbital Hematoma, visual prognosis, angio-MRI.

Copyright () 2021 The Author(s): This is an open-access article distributed under the terms of the Creative Commons Attribution 4.0 International License (CC BY-NC 4.0) which permits unrestricted use, distribution, and reproduction in any medium for non-commercial use provided the original author and source are credited.

\section{INTRODUCTION}

Spontaneous intra-orbital hematoma is a rare condition, especially in adults. It often manifests as a painful exophthalmia of rapid installation, associated with oculomotricity disorders. Neuroradiological examinations are of paramount importance for positive diagnosis. We illustrate through this clinical observation, the interest of anamnestic, clinical and radiological investigation in the etiological diagnosis of this-condition.

\section{Clinical Case}

A50-year-old woman presented with left orbital swelling and oribital diplopia after an acute episode of dry cough. The patient did not report any traumatic event and was not undergoing any anticoagulant treatment. Ophthalmological examination objectified a painful, axile, non-reducible, non-pulsatile left exophthalmia, ptosis of the left upper eyelid and total ophthalmoplegia. Visual acuity was 4/10 un improved left, 10/10 right with a normal anterior segment, including no exposure keratitis. The back of the eye showed a stage II papillary edema. The patient's CT scan showed an intra-conical and extra conical intra orbital process that repressed the optic nerve, responsible for grade II exophthalmia.

In order to eliminate a vascular origin, the patient benefited fromangio-MRI that did not objectify any vascular malformation. However, the etiological investigation revealed a chronic paroxysmal cough, without high blood pressure or blood spat disorders. This intra-orbital hematoma then occurred during a prolonged and repeated Valsalva maneuver. Evolution under symptomatic treatment was favorable with total regression of exophthalmia and ad integrum recovery of visual acuity and oculomotricity.

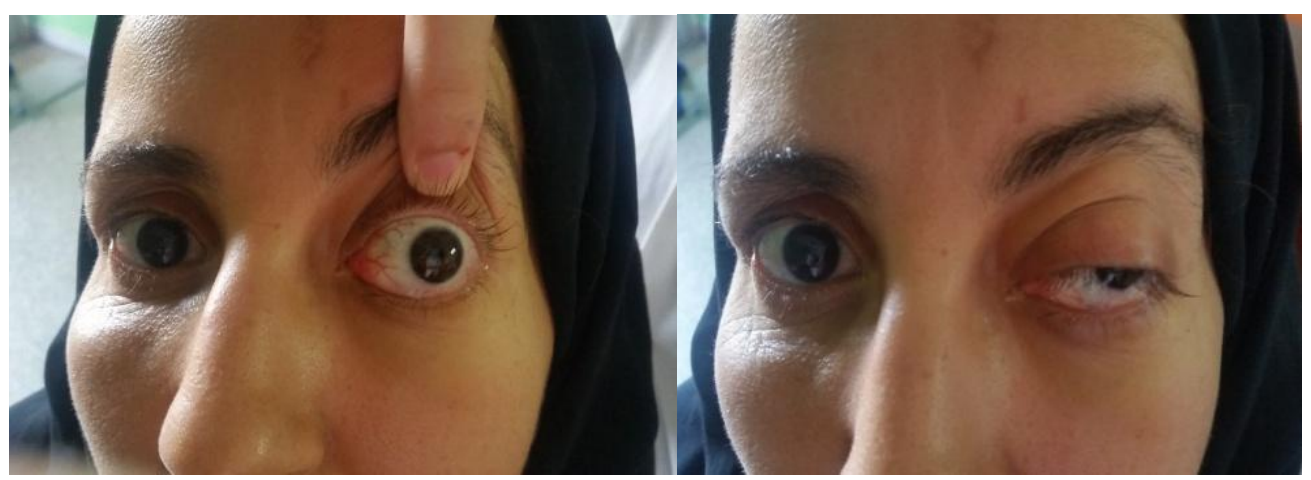

Citation: N. Inzale et al. Spontaneous Orbital Hematoma Induced by VALSAVA Maneuver. Sch J Med Case Rep, 2021 Apr 9(4): 409-411. 
Fig-1: Nonpersatile irreducible axile exophthalmia with ptosis of the left upper eyelid and total ophthalmoplegia

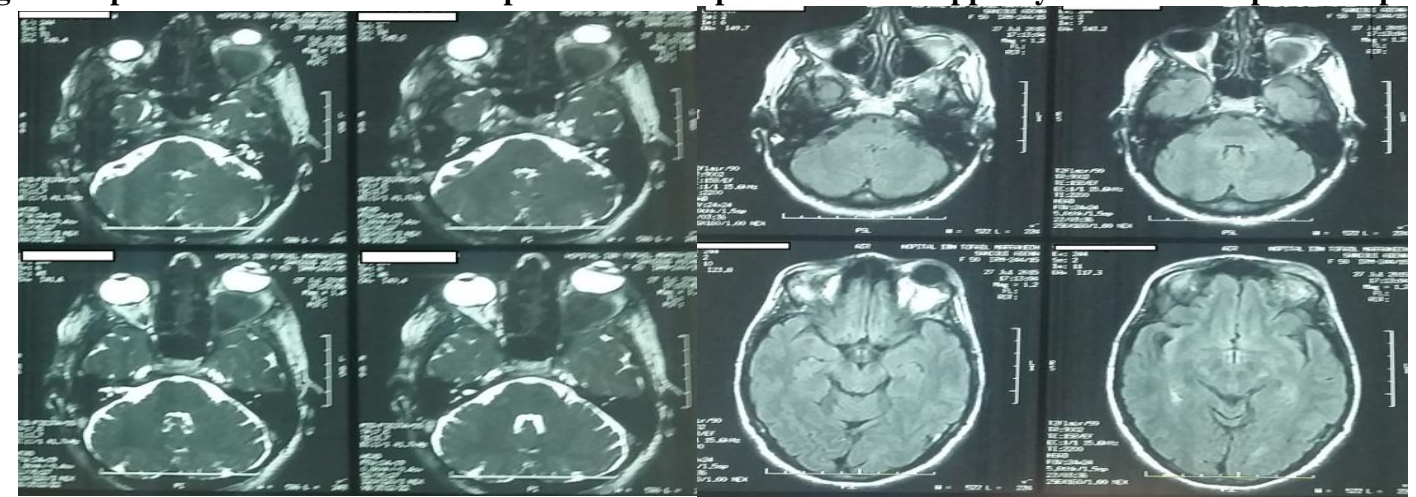

Fig-2: Oculo-orbito-cerebral computed tomography: Hyperdenseintraconic and extraconic process, not enhanced after contrast product injection, pushing the eyeball down and out. There is no other individualized lesion. This image evokes a spontaneous orbital hematoma

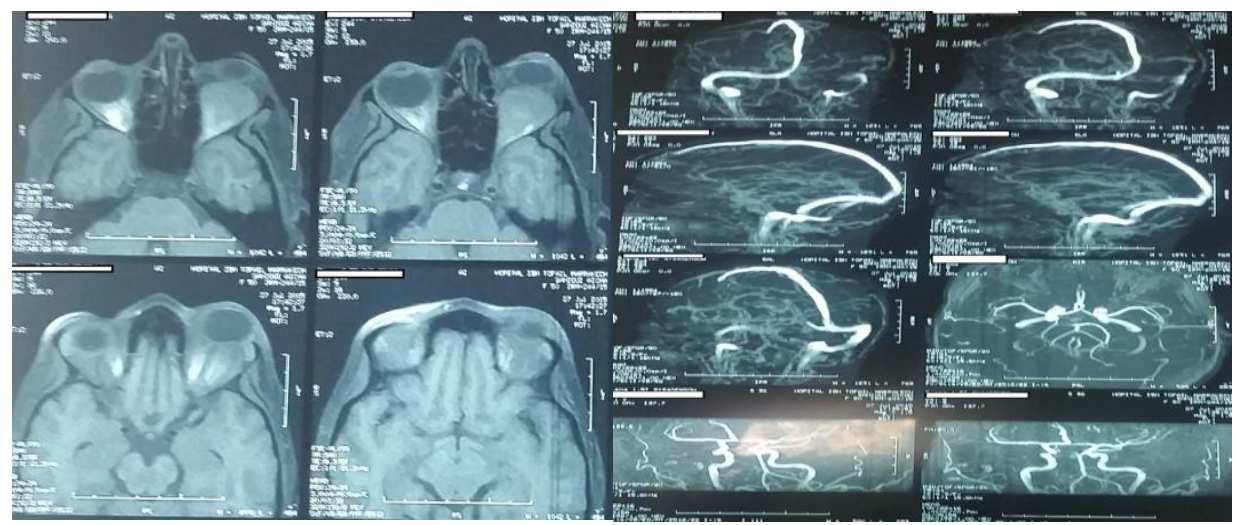

Fig-3: Magnetic resonance imaging with cerebral oulo-orbito angiographic sequence: Left intra-orbital expansive process in hyposignal on all sequences with a peripheral shell in hypersignal T1 and isosignal T2. There is no individualized vascular malformation in angiographic sequences.

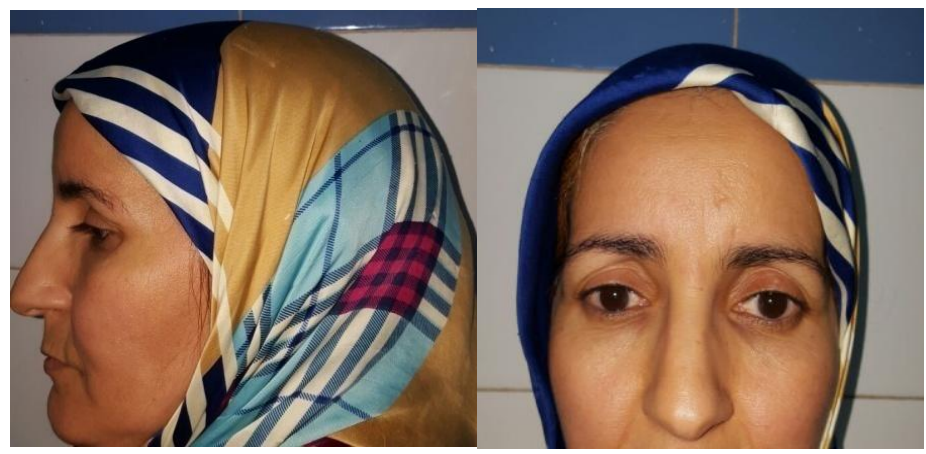

Fig-4: Total regression of exophthalmia and ad integrum recovery from visual acuity and oculomotricity

\section{DiSCUSSION}

Orbital hematomas are usually easy to diagnose, especially in the context of facial trauma or naso-sinus surgery. Outside of these contexts, their occurrence is rare, thus rendering the etiological diagnosis challenging $[1,2]$.

Clinically, intra-orbital hematoma manifests as painful exophthalmia accompanied by diplopia and oculomotor disorders depending on its location. The decrease in visual acuity can be observed when the optic nerve or central artery of the retina isare compressed [3].
The positive diagnosis of intra-orbital hematoma, outside of an evocative context, is essentially based on imaging. Spontaneous hyperdensity in the CT scans in this case was in favor of its hematic nature [4]

The rupture of vascular malformations is the first cause to be sought, thus an aneurysm of the ophthalmic artery or a venous arterial malformation was eliminated in our patient before the angiographic data. Orbital hematomas may also reveal a tumor etiology, such as lymphangiomas or cavernous angiomas, and may become complicated by an abscess when they are secondary to acute sinusitis or sinusal mucocele. In the absence of a local cause identifiable to imaging, the 
N. Inzale et al., Sch J Med Case Rep, Apr, 2021; 9(4): 409-411

search for a hematological disease such as leukemia or hemophilia should be considered $[2,4,5]$.

Valsalva's maneuver was described as a cause of conjunctiva hemorrhage, hemorrhagic retinopathy or peri-orbital petechiae. Our case reports intraoral hematoma as another ophthalmic complication of Valsalva's maneuver [6].

The treatment of intra-orbital hematomas depends on their volume and their impact. In cases of large hematomas compressing the optic nerve, emergency drainage is required, while for small-volume hematomas with no visual impact, symptomatic treatment is the rule, given the usual spontaneous resorption $[1,2]$.

\section{Conclusion}

Spontaneous intra-orbital hematoma is a rare condition, threatening visual prognosis, and should be evoked before any painful exophthalmia of sudden installation. Etiologies are diverse and positive diagnosis is based on a cluster of anamnestic and clinical arguments guided by a radiological assessment in particular MRI. Early therapeutic management is the only guarantee of a favorable outcome.
Statement of Interest: The authors state that they have no conflict of interest in relation to this article.

\section{REFERENCES}

1. Louati H, Hedhli M, Chebbi A, Hassine B, Douira W, Lahmar L, Ayed S, Bellagha I. Spontaneous orbital hematoma: Two case reports. Journal francais d'ophtalmologie. 2012 Jul 12;35(7):533e1.

2. Hajji Z, Cherqi J, Berraho A. Spontaneous orbital hematoma in an adult. A case report. J FrOphtalmol. 2004;27:267-70.

3. Kwon JH, Song YJ, Choi SS, Kim KU. Spontaneous intraorbital hemorrhage: a case report. J Korean Neurosurg Soc. 2008;44:156-8.

4. Héran F. Imaging of orbital masses. Neurosurgery. 2010;56:89-120.

5. Naggara O, Koskas P, Lafitte F, Heran F, Piekarski JD, Meder JF, Berges O. Les lésions vasculaires de l'orbite. Journal de Radiologie. 2006 Jan $1 ; 87(1): 17-27$.

6. Barrett K, Raymond C. Departments of Ophthalmology and Neuroscience, University of California, San Diego - American journal of ophthalmology. 1985. 